\title{
Metodología para la caracterización de suelos con fines de cimentación
}

\section{Methodology for soil characterization for foundation purposes}

\author{
${ }^{1}$ Condori Quispe, B.M.; ${ }^{2}$ Agüero Angulo, M. \\ Facultad de Ingeniería Civil, Universidad Nacional del Centro del Perú \\ Email: bcondori @uncp.edu.pe
}

\section{Resumen}

La investigación surge como respuesta a la problemática encontrada, como fallas, en las construcciones del distrito Tres de Diciembre, tiene como objetivo proponer una metodología para caracterizar los suelos con presencia de bolones y nivel freático, para tal caso, se analizaron 10 muestras, de suelo en estado inalterado. La investigacion obedece al diseño experimental, los ensayos se realizaron en el Laboratorio de Investigación de la Facultad de Ingeniería Civil. Luego de haber realizado el análisis y la discusión de resultados, mediante la T-studen, se llega a la conclusión que las propiedades mas influyentes para la caracterización del suelo son las propiedades físicas, mecánicas y químicas $(\mathrm{p}<0.05)$ se propone que en la clasificación de suelos sea considerado la bolonería Bo.

Palabras Clave: caracterización de suelos, cimentación, fallas de construcción, análisis de suelos

\begin{abstract}
The investigation arises as a response to the problematic found, such as constructions faults in the Tres de Diciembre district, it entails the objective of proposing a methodology to characterize the soils with the presence of bolons and groundwater table, for such case, 10 soil samples were analyzed in an unaltered state. The research follows the experimental design; the tests were carried out in the Research Laboratory of the Ingeniería Civil department. After having performed the analysis and discussion of results, the T-studen concludes that the most influential properties for soil characterization are the physical, mechanical and chemical properties $(p<0.05)$ it is proposed that in the Soil classification is considered bolonería Bo.
\end{abstract}

Keywords: characterization of soils, foundations, construction faults, soil analysis 


\section{Introducción}

La caracterización del suelo, consistió en determinar características o atributos propios de la zona andina con fines de cimentación; la falta de una metodología adecuada, ocasiona proyectos inconclusos que en muchas ocasiones son implementadas durante la ejecución de una obra el cual no es conveniente, en tal sentido, se observa, en el distrito Tres de Diciembre, fallas en las construcciones los cuales conducen al colapso que ponen en riesgo la vida humana y ocasionan pérdidas económicas.

El objetivo es proporcionar una metodología para caracterizar suelos con bolonería y presencia de nivel freático.

La investigación se inicia con los estudios geológicos, luego se determinó las propiedades físicas, como son el contenido de humedad, límites de consistencia, pesos específicos y granulometría. Estos resultados se tienen en la Tabla 1. En referencia a la granulometría, según la clasificación SUCS, se tiene SP, SC (arena mal graduadas, arenas arcillosas) también se encontró bolonería (Bo), proponiéndose que sea $\mathrm{SP}+\mathrm{Bo}$ ó $\mathrm{SC}+\mathrm{Bo}$, toda vez que no puede descartarse este material importante. En cuanto a las propiedades mecánicas, se considera los parámetros de resistencia al corte, como son el ángulo de fricción y cohesión, luego las propiedades químicas. Allí la relevancia del estudio que permitirá su caracterización de manera adecuada.

Dado los objetivos del presente estudio, los factores del medio físico, cuyo estudio resulta de mayor interés para la caracterización de la calidad de los suelos, son los climáticos, geomorfológicos, hidrográficos, geológicos, hidrogeológicos y edáficos, así como los usos del suelo (Leguey \& Rodríguez, 2006, p.8).

En la clasificación numérica, se encontraron dos grupos de suelos y sus propiedades de agrupamiento, mediante regresiones lineales se seleccionaron las propiedades distintas del suelo: ph, porcentaje de arcilla, densidad real, precipitacion mediante un uso de un mapa de suelos y retención de humedad (Bautista \& Rivas, 1998, p.21).

Para Bastidas \& Carbonell (2010), Existen diferencias para los perfiles espectrales a nivel de subgrupo taxonómico entre los 400 y 2500nm; sin embargo, para poder correlacionar la información de los perfiles espectrales con concentración de minerales arcillosos es preciso tener información cuantitativa.

Muñoz M., Muñoz C., Mancilla y Rodríguez (2007), mencionan que las arcillas son materia prima importante, para la elaboración de cerámica tradicional. Es de gran interés, por lo tanto, caracterizarlas física y químicamente para poder optimizar sus propiedades y potencializar su uso, es por esto, que la caracterización de las arcillas de la región de Guapi se torna en una actividad importante por el efecto tecnológico, económico y social que se puede generar (p.537).

Higuera, Jenny, \& Pardo (2012), proponen una metodología para el tratamiento de suelos susceptibles (arcilla) mediante la incorporación del hidróxido de calcio como estabilizante, en busca del mejoramiento de sus características físicas, mecánicas y químicas. La caracterización del suelo consistió en la elaboración de una serie de ensayos de laboratorio que determinaron sus características físico-mecánicas y químicas (p.21).

Referente al sedimento, este se caracterizó como rico en material orgánica, con zonas en donde el pH tiende a la acidez y presenta cantidades variantes de nutrientes, correspondiendo al grado de descomposición del detritus (Migdalia, Elizalde, Quiroz, \& García, 2005, p.51).

En caso de suelos granulares gruesos y densos, con presencia de bolos de grandes dimensiones, se suma la relativa representatividad de los ensayos presiométricos, la dificultad de conocer su huso granulométrico con mínima precisión, etc. Requiere del uso de técnicas menos frecuentes o habituales (Sánchez, Rodríguez, López, \& Ángel, 2016, p.305).

Dada la presencia de partículas de gran tamaño, es que se ha observado que los ensayos estándares, para obtener parámetros de resistencia y deformación, no son estables a las condiciones reales de terreno. Ante esto, MWH como ingeniero consultor, ha propuesto a Glencore una metodología de escalonamiento consistente en la extrapolación de los parámetros resistentes al rango representativo del terreno, a partir de aquellos que se obtienen desde materiales con tamaños máximos menores. Para estos efectos, se determinó una curva granulométrica, característica de terreno a partir de la cual es posible definir curvas paralelas, con tamaños máximos menores y que son fácilmente ensayables (Pérez, 2018).

Para MohammadAhmadi* (2017), el suelo seco, natural y sin cohesión, raramente se encuentra en áreas urbanas; sin embargo, estudios previos sobre la propagación de la fractura superficial usando modelos físicos, han concentrado habitualmente el suelo sin cohesión.

En Xi1, Assessing the effect of potential water and salt intrusion on coastal wetland soil quality: simulation study (15 december 2018), los resultados mostraron que demasiada agua y sal en el suelo puede disminuir la calidad del suelo, y el efecto del agua fue más obvio que el salino.

La técnica de inyección de la carcasa del conductor se ha aplicado cada vez más en la perforación en aguas profundas. El proceso de excavación continua en tiempo real se simula con el método multifásico de volumen de líquido (VOF) del software de cálculo de flui- 
dos ANSYS CFD (dinámica de fluidos computacional) en el estudio actual. El suelo cohesivo se modela utilizando un tipo de fluido viscoso con el modelo Herschel-Bulkley. (TengWang*, 2019), Xian, Pang, Zhang, et al., (2019), en el estudio de ellos, se diseñó un experimento de simulación para identificar el impacto del agua y la sal en la calidad del suelo en el humedal costero de la bahía de Jiaozhou, China.

Hernández-Acosta \& Gutiérrez-Castorena (2006), manifiestan que uno de los primeros trabajos a realizarse son el análisis químico, físico y biológico. En el trabajo se seleccionaron al azar nueve puntos de muestreo, se realizó el perfil de su morfología. Las fracciones predominantes fueron las propiedades químicas y físicas, el contenido de materia orgánica de pobre a media y un bajo contenido de sales. Los primeros estudios en suelos contaminados que se quiere remediar fueron los análisis químico, físico y biológico.

En la investigación de Sue Wei-Salas (2014), fue con el objetivo de aportar para el ordenamiento y planificación del uso, se utilizaron imágenes aéreas y la información fue procesada por medio del programa de computo ArgGIS9.2. Las imágenes, permitieron identificar la distribución y extensión de los principales cultivos.

La caracterización inicial permitió identificar la necesidad de realización de campañas de muestreo. Tiene como objeto determinar la calidad pre operacional del suelo como base de comparación futura, en emplazamientos que puedan albergar actividades potencialmente contaminantes (Urbanística, 2003).

En el estudio de Olarte \& Quevedo (s/f), se han reconocido, en la zona 19 , unidades de suelos a nivel taxonómico de la eate boria de subgrupos del sistema, suelos desarrollados a partir de materiales de aportes fluviales recientes, distribuyéndose a lo largo de los ríos, todo perfil es moderadamente calcáreo.

\section{Materiales y métodos}

\section{Materiales}

El distrito Tres de Diciembre, lugar de escasos recursos, se ubica en la provincia de Chupaca, región Junín a 2400msnm, la zona esta caracterizada por extensas áreas con presencia de nivel freático, suelos finos en su mayoría, zona dedicada a la agricultura y, en menor escala, a la ganadería. La población estudiada fue de $3 \mathrm{Ha}$.

El enfoque de la investigación es cuantitativo, el método deductivo mide valores cuantificables; y el nivel es el explicativo.

Las 10 muestras obtenidas fueron analizadas en el Laboratorio de Mecánica de Suelos de la FIC/UNCP. Se conocieron las propiedades físicas como el contenido de humedad del suelo, pesos específicos volumétricos, gravedades específicas y granulometría. Así mismo, se conocieron los parámetros de resistencia al corte, como fueron la cohesión y el ángulo de fricción.

Para el proceso cuantitativo la muestra es un subgrupo de la población de interés sobre el cual se recolectarán datos, este deberá ser representativo de dicha población (Hernández \& Fernández, 2010, p.173).

\section{Técnicas}

Según Naupas (2013), las técnicas pueden definirse como un conjunto de normas que regulan el proceso de investigación, en cada etapa, desde el principio hasta el fin; desde el descubrimiento del problema hasta la verificación e incorporación de las hipótesis, dentro de las teorías vigentes. Son parte del método científico. Según Rodríguez (1986), se clasifican en conceptuales, descriptivas y cuantitativas (p.135).

Durante el desarrollo del trabajo se desarrollaron técnicas matemáticas-estadísticas.

En el laboratorio, se utilizaron formatos para la obtención de resultados; asimismo, los procedimientos fueron de acuerdo con las normas ASTM y NTP. Se trabajaron con muestras inalteradas y alteradas, fueron 30 observaciones para 10 muestras.

Los instrumentos utilizados fueron el microscopio, equipo triaxial (Figura 1), corte directo, tamices, libreta de apuntes y; asimismo, otros misceláneos.

Figura 1

Equipo triaxial utilizado para determinar las propiedades mecánicas

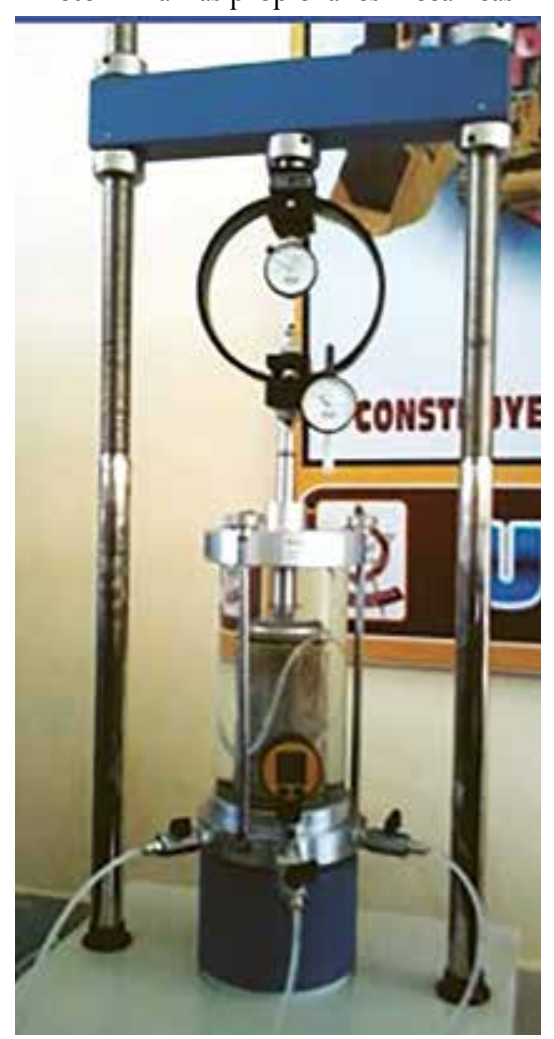




\section{Figura 2}

Colocación de la membrana de hule en la muestra de suelo en el equipo triaxial

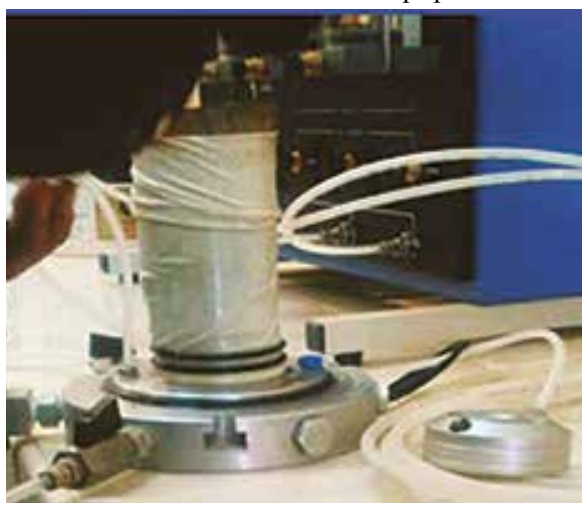

\section{Ensayos}

- Peso específico volumétrico, realizada de acuerdo a la Norma Técnica Peruana, tanto para suelos cohesivos, como granulares.

- Granulometría y análisis granulométricos por tamizado (NTP 339.128/ASTM D 422).

- Límite líquido y plástico (NTP 339.129/ASTM D 4318).

- Para el análisis granulométrico y clasificación de suelos, es necesario considerar la bolonería o partículas superiores a las gravas, como se puede observar en la Tabla 3.

- Contenido de humedad, el procedimiento es desarrollado de acuerdo a las NTP 339.127/ASTM D 2216.

- Propiedades químicas, se han considerado al contenido de sales solubles totales en suelos y agua subterránea (NTP 339.152/BS 1377), contenido de cloruros solubles en suelos y agua subterránea (NTP 339.177/AASHTO T 291) y al contenido de sulfatos solubles en suelos y agua subterránea (NTP 339.178/AASHTO T 290).

- Triaxial, el equipo fue utilizado para la determinación de las propiedades mecánicas como son el ángulo de fricción y la cohesión, el procedimiento fue de acuerdo a las NTP.

\section{Figura 3}

Tamices o cribas para el análisis granulométrico.

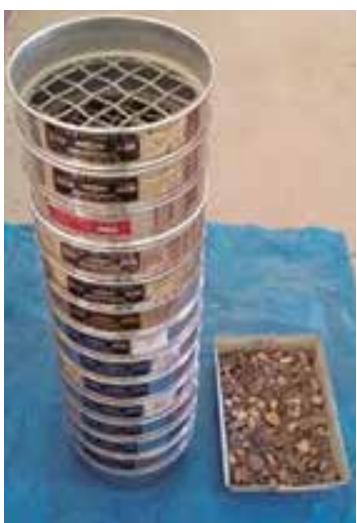

\section{Resultados y discusión}

Los resultados de las propiedades físicas y mecánicas se muestra en la Tabla 1.

Tabla 1

Propiedades físicas y mecánicas.

\begin{tabular}{ccc}
\hline \multicolumn{3}{c}{ Componentes } \\
\hline $\mathbf{W}(\%)$ & p.e $\left(\mathbf{T n} / \mathbf{m}^{3}\right)$ & $\varnothing\left({ }^{\circ}\right)$ \\
\hline 19.1 & 1.78 & 20 \\
20.7 & 1.71 & 18 \\
21 & 1.69 & 17 \\
22 & 1.69 & 17 \\
24.2 & 1.62 & 16 \\
25 & 1.62 & 14 \\
25.5 & 1.58 & 14 \\
27 & 1.5 & 12 \\
27.2 & 1.49 & 10 \\
28 & 1.49 & 10 \\
\hline
\end{tabular}

En la Figura 4, se muestra que la influencia del contenido de humedad en el ángulo de fricción disminuye al incrementarse la humedad del suelo.

Figura 4

Histograma contenido de humedad vs ángulo de fricción Ángulo de fricción vs contenido de humedad

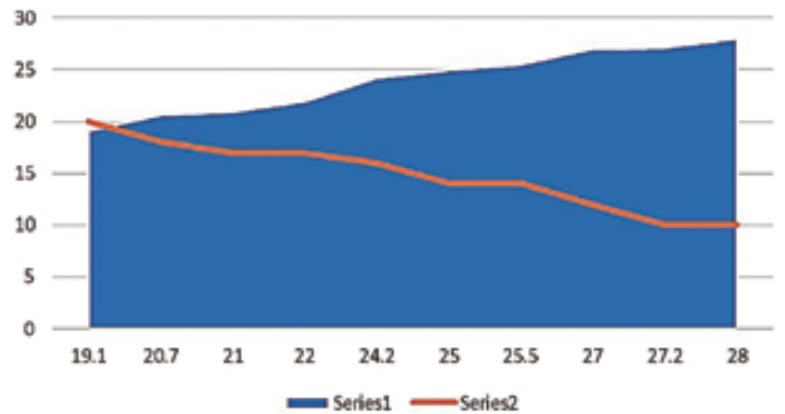

En la Figura 2, se tiene los pesos específicos volumétrico y la variación en relación con el contenido de humedad.

Figura 5

Contenido de humedad vs peso específico volumétrico

PESO ESPECÍFICO VS CONTENIDO DE HUMEDAD

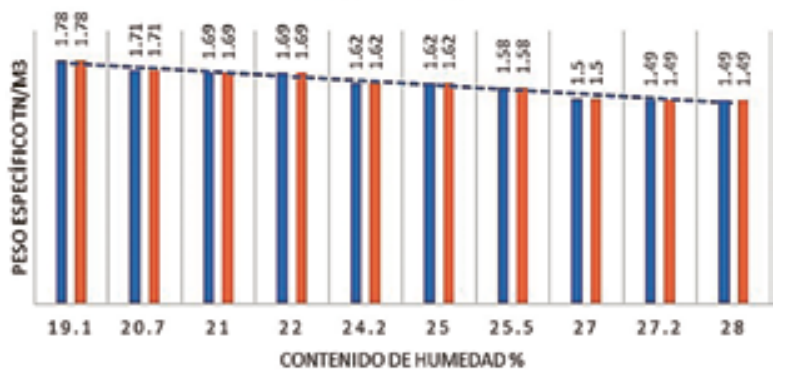


En cuanto a las propiedades químicas, se obtuvo $\mathrm{pH}$ superiores a 7 , consideradas como soluciones básicas. El pH, midió la acidez o alcalinidad de una disolución; en la Tabla 2, se tiene valores de $\mathrm{Ca}, \mathrm{Mg}, \mathrm{K}, \mathrm{Na}$ (cationes).

Tabla 2

Propiedades químicas

\begin{tabular}{ccccc}
\hline $\mathbf{C a}$ & $\mathbf{M g}$ & $\mathbf{K}$ & $\mathbf{N a}$ & $\mathbf{P h}$ \\
\hline 24.8 & 8.23 & 0.17 & 0.11 & 7.63 \\
\hline 18.08 & 5.65 & 0.35 & 0.08 & 7.53 \\
\hline
\end{tabular}

Tabla 3

Clasificación de suelos

\begin{tabular}{ccc}
\hline $\begin{array}{c}\text { Clasificación } \\
\text { SUCS }\end{array}$ & Material presente & Propuesta \\
\hline SC & Bo & SC+Bo \\
SP & Bo & SP+Bo \\
\hline
\end{tabular}

El contenido de humedad es una propiedad física del suelo; para este caso, la más influyente. Los valores encontrados fueron de $19 \%$ al $28 \%$ considerado como humedades medias para una época de sequía, estos valores influyen en el comportamiento del suelo como es el caso de los desplazamientos; asimismo, en otras propiedades, como las químicas y mecánicas.

En épocas de lluvia los contenidos de humedad alcanzan hasta el $38 \%$, el valor de la cohesión disminuye considerablemente. Leguey \& Rodríguez (2006), indican que los factores climáticos, geomorfológicos, uso de suelo y otros, resultan ser importantes para la caracterización.

Figura 6

Curva esfuerzo- deformación

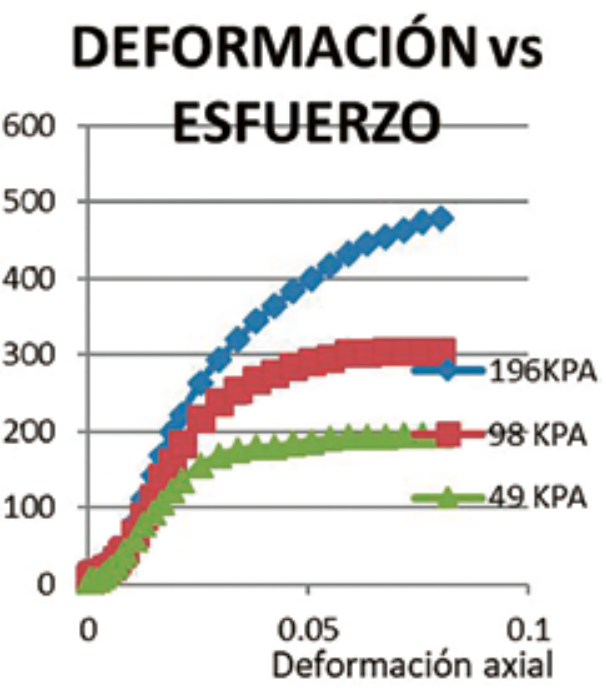

\section{Conclusiones}

- La eficiencia de la fitorremediación del cobre en una solución acuosa con la especie lenteja de agua (Lemna minnor) se evaluó hasta un 68,57 \% de remoción.

\section{Referencias bibliográficas}

Bastidas, E., \& Carbonell, J. (2010). Caracterización espectral y mineralógica de los suelos del valle del río Cauca por espectroscopía visible e infrarroja (400-2.500nm). Red de revistas científicas de América Latina y El Caribe, España y Portugal, 291.

Bautista, F., \& Rivas, H. (1998). Caracterización y clasificación de suelos con fines productivos en Córdoba, Veracruz, México. México.

Hernández, R., \& Fernández, C. (2010). Metodología de la investigación. México: Mc Graw Hill.

Hernández-Acosta, E., \& Gutierrez-Castorena, M. d. (2006). Caracterización del suelo y plantas de un sitio contaminado con hidrocarburos. Terra Latinoamericana, 463-470.

Higuera, C., Jenny, G., \& Pardo, O. (2012). Caracterización de un suelo arcilloso tratado con hidróxido de calcio. Revista ingeniería, 21-40.

Leguey, M., \& Rodríguez, M. (2006). Plan de sectorización las laderas - Peñas Blancas. Madrid: Argongra, S.L.

Migdalia, D.-V., Elizalde, E., Quiroz, H., \& Garcia, J. (2005). Caracterización de algunos parámetros físico químicos del agua y sedimento del lago Zempoala, Morelos, México. Red de revistas científicas de América Latina y el Caribe, España y Portugal., 57-65.

MohammadAhmadi*, M. (2017). Experimental investigation of reverse fault rupture propagation through cohesive granular soils. Elsevier.

Muñoz Meneses R. A., Muñoz Cháves J. A., Mancilla P., Rodríguez Páez J. E. (2007). Caracterización físicoquímica de arcillas del municipio de Guapi-Costa Pacífica Caucana. Revista de la académia Colombiana de ciencias exactas, físicas y naturales, 537-544.

Naupas, H. (2013). Metodología de la investigación. Colombia: Ediciones de la U.

Olarte, C., \& Quevedo, R. (s.f.). Caracterización y evaluación de la capacidad de uso de los suelos de la coliñarca de El Aljarafe. CEBAC.

Pérez, G. (2018). Caracterización de suelos granulares gruesos. IOS Press Ebooks, 1423-1431.

Sánchez, S., Rodríguez, J., López, J. G., \& Ángel, J. (2016). Caracterización de suelos granulares gruesos. $10^{\circ}$ Simposio Nacional de Ingeniería geotécnica, 305312. 
Sue Wei-Salas, A. (2014). Caracterización del uso del suelo en las principales áreas agrícolas de la gran área metropolitana de Costa Rica. Sistemas de información geográfica.

Urbanística, C. g. (2003). Caracterización del suelo, avance del plan de sectorización "Las laderas-Peñas Blancas".

Xian, X., Pang, M., Zhang, J. et al. Assessing the effect of potential water and salt intrusion on coastal wetland soil quality: simulation study. J Soils Sediments 19, 2251-2264 (2019). https://doi. org/10.1007/s11368-018-02225-y 